www. revistadyo.com

\title{
Los recursos y capacidades en la adopción de una estrategia de mercadotecnia verde: evidencia en hoteles de turismo de sol y playa de Oaxaca, México
}

\author{
Eliomar Hernández-García, Diana de Yta-Castillo y Patricia S. Sánchez-Medina
}

Recibido: 16 de Febrero de 2020

Aceptado: 4 de Abril de 2020

https://doi.org/10.37610/dyo.v0i72.585

\section{Resumen}

A partir de la Teoría basada en los Recursos (TBR), en este artículo se estudia cómo los recursos y las capacidades contribuyen en la adopción de una estrategia de mercadotecnia verde (EMV) en 73 hoteles de tres, cuatro y cinco estrellas de Bahías de Huatulco y Puerto Escondido, Oaxaca, México. Los hallazgos demuestran que los recursos (físicos, financieros y humanos) y las capacidades (visión compartida y gestión de los grupos de interés) permiten adoptar una estrategia de mercadotecnia verde y competir a través de la comercialización de productos y servicios más amigables con el medio ambiente. Para la obtención de los datos se estratificó la muestra en tipo de alojamiento turístico y número de estrellas y se utilizó un cuestionario estructurado. Los resultados obtenidos son de utilidad al sector hotelero, las instituciones públicas y privadas encargadas del turismo como Asociaciones de Hoteles y Moteles y Secretarias de Turismo, ya que proporciona información relevante sobre los recursos y capacidades que la empresa puede utilizar para el despliegue de la estrategia de mercadotecnia verde. A la fecha, se han realizado pocos estudios empíricos que analicen el tema en cuestión, por lo cual este trabajo contribuye a la TBR debido a que las investigaciones sobre la estrategia de mercadotecnia verde se enfocan más en los efectos que tiene ésta y no en los factores que conducen a su desarrollo, lo que ha dejado un hueco en la literatura sobre los recursos y las capacidades de la empresa que contribuyen a una EMV.

\section{Palabras clave}

Estrategia de mercadotecnia verde, industria turística, hoteles de sol y playa, teoría basada en los recursos.

\section{Introducción}

La TBR señala que el éxito de una empresa depende de sus recursos y capacidades internas (Barney, 1991; Aragón y Sharma, 2003; Fong, 2011; Hart, 1995). En la literatura académica, la TBR se ha empleado con regularidad para estudiar la influencia de los recursos y capacidades para adoptar estrategias ambientales en las empresas (AragónCorrea et al., 2005; Garay y Font, 2012; Menguc et al., 2010; Torugsa et al., 2012). Recientemente, son los clientes, reguladores, entre otros grupos de interés, quienes ejercen mayor presión sobre las organizaciones para llevar a cabo

Eliomar Hernández-García *

iD https://orcid.org/0000-0003-1694-0251

Diana de Yta-Castillo **

ddeyta@conacyt.mx

iD https://orcid.org/0000-0002-5317-1343

Patricia S. Sánchez-Medina ***

psanchez@ipn.mx

* Universidad del Mar Campus Huatulco de Oaxaca, México.

** CONACYT. Universidad Nacional Autónoma de México

*** Instituto Politécnico Nacional (CIIDIR-IPN Unidad Oaxaca), México. acciones ambientales. Por lo que, los negocios han incluido los temas ecológicos en sus estrategias empresariales (Plaza et al., 2011; Sarkis et al., 2010), incluyendo su estrategia de mercadotecnia. En la actualidad, diversas empresas se han desplazado de una estrategia de mercadotecnia tradicionalhacia una EMV y, por ello, sus mezclas de mercadotecnia (producto, precio, plaza y promoción) se basan en criterios verdes.

Los hoteles son empresas particularmente importantes porque se han vuelto la cara de la empresa turística y porque están estrechamente relacionados con el medio ambiente (Vargas et al., 2011). Los hoteles necesitan un medio ambiente sano para existir y a la vez, generan fuertes impactos ambientales a través del alto consumo de agua y energía eléctrica, entre otros efectos en el entorno natural (Radwan et al., 2012; Reyes-Santiago y Sánchez-Medina, 2016). Al mismo tiempo, los hoteles tratan de minimizar estos impactos a través de diversas acciones verdes en su mezcla de mercadotecnia. En los productos/servicios, se puede notar en la adopción de productos amigables con el entorno natural, compra de productos orgánicos, menor 
consumo de recursos y, en el reuso y/o reciclaje de recursos (de Yta-Castillo y Sánchez-Medina, 2017; de Yta-Castillo et al., 2018; Kasim et al., 2014; Velázquez-Castro et al., 2016); en el precio, es posible advertirlo cuando los hoteles ofrecen tarifas competitivas y/o pueden cobrar un precio Premium porque su servicio conlleva iniciativas que contribuyen a impactar en menor medida el medio ambiente (Leonidou et al. 2013a; Rivera, 2002). En la dimensión plaza, es posible observarlo cuando los hoteles eligen proveedores que muestran responsabilidad ambiental (Teng et al., 2012) y, finalmente, en la promoción, se advierte cuando los hoteles muestran cooperación y comunicación con sus empleados y clientes sobre temas ambientales (de Yta-Castillo y SánchezMedina, 2017; Leonidou et al., 2013a; Teng et al. 2012) y cuando promueven sus iniciativas ambientales en sus páginas de internet y redes sociales (Chan, 2013).

Sin embargo, como lo señala Tiwari et al. (2011), la mercadotecnia verde aún está en su infancia. En el sector hotelero, la mayor parte de los estudios se han centrado en las preferencias, intenciones y percepciones de los huéspedes (Chan, 2014). Por otro lado, los estudios de mercadotecnia verde en empresas son pocos y se han realizado en la industria manufacturera (Chan, 2014). Por lo que, se necesitan estudios que analicen la percepción de los gerentes del sector servicios acerca de la EMV, particularmente, la percepción de los gerentes del sector hotelero (Chan, 2013; El Dief y Font, 2010). En particular, se requieren investigaciones que analicen el rol de los recursos y capacidades en la adopción de una EMV en el sector hotelero (Leonidou y Leonidou, 2011; Leonidou et al., 2013a).

En México se han realizado investigaciones que abordan los temas ambientales en el sector hotelero. SánchezMedina et al. (2016) realizaron una investigación en hoteles del estado de Oaxaca, en donde analizan la relación entre los intereses de los stakeholders y la implementación de prácticas de gestión ambiental y cómo la antigüedad, tamaño y capacidad económica del hotel son variables que moderan dicha relación. Ellos encontraron que los intereses de los stakeholders tienen un impacto positivo en el desarrollo de prácticas ambientales y que la antigüedad y el tamaño son variables que moderan dicha relación. Reyes-Santiago et al. (2017) analizaron la relación entre cultura organizacional y eco-innovación en hoteles del estado de Oaxaca. Ellos encontraron que la cultura adhocrática y de clan tienen una relación positiva con la implementación de eco-innovaciones. de Yta-Castillo et al. (2018) analizaron la relación entre compromiso ambiental, desempeño ambiental y desempeño en hoteles de cinco estados de la República Mexicana. Ellos encontraron que el compromiso ambiental se relaciona de manera positiva con el desempeño ambiental y que éste tiene una relación positiva y no significativa con el desempeño del hotel. Finalmente, Reyes-Santiago et al. (2019) analizaron la relación entre una estrategia ambiental proactiva y la eco-innovación y la relación de estas dos capacidades dinámicas con el desempeño ambiental y organizacional de hoteles del estado de Oaxaca. Ellos encontraron que la estrategia ambiental se relaciona positivamente con la eco-innovación, el desempeño ambiental y organizacional. Asimismo, ellos hallaron que la eco-innovación se relaciona positivamente con el desempeño ambiental y negativamente con el desempeño organizacional. Como se observa, en México existen estudios que abordan la gestión ambiental en los hoteles; sin embargo, no se han realizado estudios que aborden la adopción de una EMV tomando como base la posesión de recursos y capacidades, como lo plantea la TBR. Por lo tanto, este estudio propone analizar el efecto de los recursos y capacidades en la adopción de una EMV en hoteles de turismo de sol y playa de Bahías de Huatulco y Puerto Escondido en Oaxaca, México.

Para la economía mexicana, el turismo es uno de sus principales motores debido a que ocupa el tercer lugar dentro de las principales actividades generadoras de divisas (Cruz et al., 2014). En México, el estado de Oaxaca es una entidad destacada a nivel mundial por sus atractivos naturales y culturales (Cruz-Bautista, 2013; Reyes-Santiago et al., 2019) y con fuerte vocación turística. En Oaxaca, los dos destinos turísticos de sol y playa más importantes son Bahías de Huatulco y Puerto Escondido (SECTUR, 2016).

Derivado de lo anterior, este estudio contribuye a: 1) la TBR al estudiar la influencia de los recursos y capacidades en la adopción de una EMV en el sector hotelero; 2) llenar el vacío en la literatura académica al examinar una EMV desde la percepción de los gerentes, particularmente de empresas hoteleras; 3) la literatura académica sobre mercadotecnia verde.

Este documento está organizado como sigue: la sección 2 presenta la revisión de la literatura y el desarrollo de las hipótesis; la sección 3 presenta la metodología, las medidas y el tratamiento de las variables; la sección 4 explica los resultados y las discusiones $\mathrm{y}$, finalmente, la sección 5 presenta las conclusiones del estudio.

\section{Revisión de la literatura y desarrollo de hipótesis}

\section{Teoría basada en los recursos}

La TBR explica que la ventaja competitiva de una empresa depende de sus aptitudes y capacidades intrínsecas y no del análisis del medio ambiente competitivo (Barney, 1991). Para esta teoría, cada empresa tiene recursos y capacidades internas que le permiten desarrollar estrategias empresariales que, a su vez le van a posibilitar obtener una ventaja competitiva y mejorar su desempeño (Barney, 1991; Le et al., 2006; Yin y Schmeidler, 2009).

Por un lado, los recursos son insumos en el proceso de producción (Grant, 1991) y se pueden clasificar como recursos de capital físico, de capital humano y de capital organizacional; pueden ser tangibles e intangibles y 
no siempre existen mercados en los cuales se puedan intercambiar (Barney, 1991). Por su parte, las capacidades son el resultado de trabajar con rutinas y recursos (Day, 1994; Grant, 1991; Hart y Dowell, 2011). En otras palabras, los recursos van a ser productivos cuando la empresa logre articularlos, integrarlos y manejarlos, de tal manera que logre crear capacidades que le permitan hacer frente al entorno externo y a la demanda cambiante (Eisenhardt y Martin, 2000).

Con la finalidad de que los recursos y capacidades sean capaces de generar una ventaja competitiva, deben poseer las siguientes características: 1) ser imperfectamente imitables por los competidores (Barney, 1991; Dierickx y Cool, 1989); 2) insustituibles (Barney, 1991; Dierickx y Cool, 1989); raros o específicos (Barney, 1986, 1991; Reed y Defillippi, 1990); inamovibles entre negocios (Barney, 1991; Conner, 1991) y valiosos (Barney, 1986, 1991).

En la literatura académica a menudo se emplea la TBR para estudiar la influencia de los recursos y capacidades en el desarrollo de estrategias ambientales y de mercadotecnia verde en las empresas (Aragón-Correa et al., 2005; LópezGamero et al., 2011; Menguc et al., 2010; Torugsa et al., 2012). Por ejemplo, López-Gamero et al. (2011) analizaron la relación entre los recursos y capacidades y la percepción de los gerentes sobre el medio ambiente como una oportunidad competitiva, en hoteles de España. Por su parte, Torugsa et al. (2012) examinaron empíricamente la relación entre las capacidades de visión compartida, gestión de los grupos de interés y proactividad estratégica, la responsabilidad social corporativa proactiva y el desempeño económico en empresas manufactureras de Australia. Por último, Leonidou et al. (2013a) analizaron cómo los recursos físicos, financieros y experienciales, las capacidades de visión compartida, construcción de relaciones y la habilidad de responder rápidamente a los cambios tecnológicos, conducen a una EMV en hoteles de Grecia. Como puede notarse, la TBR ha sido una teoría útil para analizar cómo diferentes recursos y capacidades pueden generar una estrategia empresarial que considera las restricciones ambientales y puede generar ventajas competitivas.

Además, autores como Hart (1995), Christmann (2000) y López-Gamero et al. (2009) consideran que la TBR es un marco teórico idóneo para analizar los temas ecológicos en la empresa.

\section{Recursos y EMV en hoteles}

Los recursos son insumos en el proceso de producción que permiten a la empresa desarrollar estrategias empresariales (Barney, 1991; Grant, 1991). La mercadotecnia verde forma parte de la estrategia global de la empresa (Prakash, 2002), por lo que los recursos son insumos que pueden contribuir a obtener una EMV.
En la literatura académica es posible notar que las empresas pueden desarrollar una EMV y obtener una ventaja competitiva que mejore sus resultados empresariales a través de la utilización de sus recursos (Hart, 1995; Leonidou et al., 2013a). Diversos autores (Gavronski et al., 2011; Leonidou et al., 2013a; Paulraj; 2011; Russo y Fouts, 1997; Shah, 2011) han identificado que los recursos físicos, financieros y humanos desempeñan un papel importante en el desarrollo de una EMV.

Las empresas que utilizan apropiadamente sus recursos físicos como maquinaria, equipo, instalaciones y tecnología física pueden mejorar sus prácticas ambientales en términos de reducción del desperdicio, reciclado de materiales, conservación de la energía y prevención de la contaminación (Gavronski et al., 2011; Leonidou et al., 2013a; Russo y Fouts, 1997). En los hoteles, los recursos físicos también apoyan una EMV porque se pueden generar productos/servicios más verdes al disminuirse el uso de recursos naturales y hacer frente al cambio climático (Medina-Serrano et al. 2019; Vargas, 2015). El uso de aparatos eléctricos eficientes o de energías alternativas y la infraestructura para reciclar contribuye a una mayor eficiencia en el consumo de recursos naturales en estos negocios (Velázquez-Castro, 2016). Los recursos financieros representan el financiamiento de la EMV, por lo cual permiten la compra e instalación de equipos tecnológicos más avanzados para prevenir la contaminación y atender los problemas ambientales (Chan, 2005; Leonidou et al., 2013a). El financiamiento apoya la EMV en los hoteles porque la mayoría de tecnologías que ahorran agua y electricidad requieren de grandes inversiones (VelázquezCastro et al., 2016).

Los recursos humanos son determinantes para implementar exitosamente las tecnologías verdes, generar ideas para realizar acciones ambientales e introducir valores ecológicos en la empresa (Carmona-Moreno et al., 2012; Surroca et al., 2010; Vidal et al., 2012). Los hoteles tienen muchas fuentes de contaminación debido a que su funcionamiento se basa en muchas actividades pequeñas, cada una de las cuales consume relativamente pequeñas cantidades de energía, agua, comida, papel y otros recursos (Armas-Cruz, 2011; Carmona et al., 2004; Céspedes y de Burgos, 2004; Kirk, 1995). Por lo cual, la participación del personal en cada una de estas fuentes de contaminación es importante para lograr una estrategia de mercadotecnia medioambientalmente responsable.

Desde la TBR se han identificado los recursos físicos y financieros como importantes para la empresa en la formulación de la estrategia de mercadotecnia verde (Leonidou et al., 2013a), al igual que los recursos humanos que impulsan la adopción de esta estrategia y mejoran su comportamiento ambiental (Aragón y Sharma, 2003; Surroca et al., 2010; Vidal et al., 2012). Dicho lo anterior, es pertinente estudiar el efecto que tienen los recursos físicos, financieros y humanos en la adopción de una EMV, ya que 
como lo señalan diversos autores (Gavronski et al., 2011; Leonidou et al., 2013a; Paulraj, 2011; Russo y Fouts, 1997; Shah, 2011) son elementos importantes que conllevan a un mejoramiento medioambiental y que además claramente son identificables en el contexto hotelero (Medina-Serrano et al. 2019; Vargas, 2015; Velázquez-Castro et al., 2016 ) como parte esencial para que este tipo de empresas puedan alcanzar una mejora en estrategias de mercadotecnia verde.

Con base en los argumentos anteriores, se plantean las siguientes hipótesis:

H1a: Existe una relación directa y positiva entre la posesión de recursos físicos y la adopción de una EMV

H1b: Existe una relación directa y positiva entre la posesión de recursos financieros y la adopción de una EMV

H1c: Existe una relación directa y positiva entre la posesión de recursos humanos y la adopción de una EMV

\section{Capacidades y EMV en hoteles}

La adopción de una EMV en una empresa también se deriva del desarrollo de capacidades, las cuales son resultado de integrar y desarrollar con eficacia y eficiencia los recursos únicos y heterogéneos (Day, 1994; Torugsa et al., 2012). En la literatura académica se ha encontrado que la capacidad de visión compartida, la capacidad de gestión de los grupos de interés y la capacidad de innovación continua son importantes en la adopción de una estrategia ambiental (Bianchi y Noci, 1998; González-Benito y González-Benito, 2005; Hart, 1995; Leonidou et al., 2013a; Rangone, 1999; Sharma y Vredenburg, 1998; Sharma et al., 2007; Torugsa et al., 2012).

Los hoteles que 1) comparten los temas verdes con sus empleados, pueden generar un compromiso colectivo y contribuir al éxito de la EMV (Chan, 2013); 2) cooperen y se comuniquen con diferentes grupos de interés en relación al tema medioambiental (de Yta-Castillo y Sánchez-Medina, 2017; Fraj et al., 2015; Rivera, 2002) podrían generar una mezcla de mercadotecnia que se base en criterios verdes; 3 ) innoven y/o tengan mayor apertura a innovar, son quienes tomarán la decisión de adoptar una EMV y podrán enfrentar los cambios que les exijan sus mercados para reducir ineficiencias en el uso de los recursos naturales (Le et al., 2006; Fraj et al., 2015).
Algo importante a señalar es que la creación de valor en las estrategias de mercadotecnia verde no sólo se deriva de la adquisición de recursos únicos y heterogéneos sino también de la capacidad de integrar y desplegar esos recursos como base para capacidades organizacionales (Torugsa et al., 2012). Para dar mayor información que permita el entendimiento de una estrategia de mercadotecnia verde es necesario estudiar no solo los recursos sino también las capacidades que podrían estar incentivando el desarrollo de una EMV en el contexto turístico de Bahías de Huatulco y Puerto Escondido. La literatura señala que son tres las capacidades más importantes en la adopción de una EMV: la capacidad de visión compartida, de gestión de los grupos de interés y de innovación continua (Bianchi y Noci, 1998; GonzálezBenito y González-Benito, 2005; Hart, 1995; Leonidou et al., 2013a; Rangone, 1999; Sharma y Vredenburg, 1998; Sharma et al., 2007; Torugsa et al., 2012). Además, estas capacidades también se encuentran presentes en el contexto hotelero como lo han señalado Chan (2013), de Yta-Castillo y Sánchez-Medina (2017), Fraj et al., (2015) y Rivera (2002).

Con base en los argumentos anteriores, se plantean las siguientes hipótesis:

H2a: Existe una relación directa y positiva entre la posesión de la capacidad de visión compartida y la adopción de una EMV

H2b: Existe una relación directa y positiva entre la posesión de la capacidad de gestión de los grupos de interés y la adopción de una EMV

H2c: Existe una relación directa y positiva entre la posesión de la capacidad de innovación continua y la adopción de una EMV

\section{Modelo teórico}

Desde la TBR, se propone el siguiente modelo teórico de investigación en el cual se analiza el efecto de los recursos físicos, financieros y humanos, y capacidades de visión compartida, gestión de los grupos de interés e innovación continua en la adopción de una EMV en hoteles de sol y playa de Bahías de Huatulco y Puerto Escondido, Oaxaca, México (Figura 1). 
Figura 1 Modelo teórico. Fuente: Elaboración propia con base en la TBR.

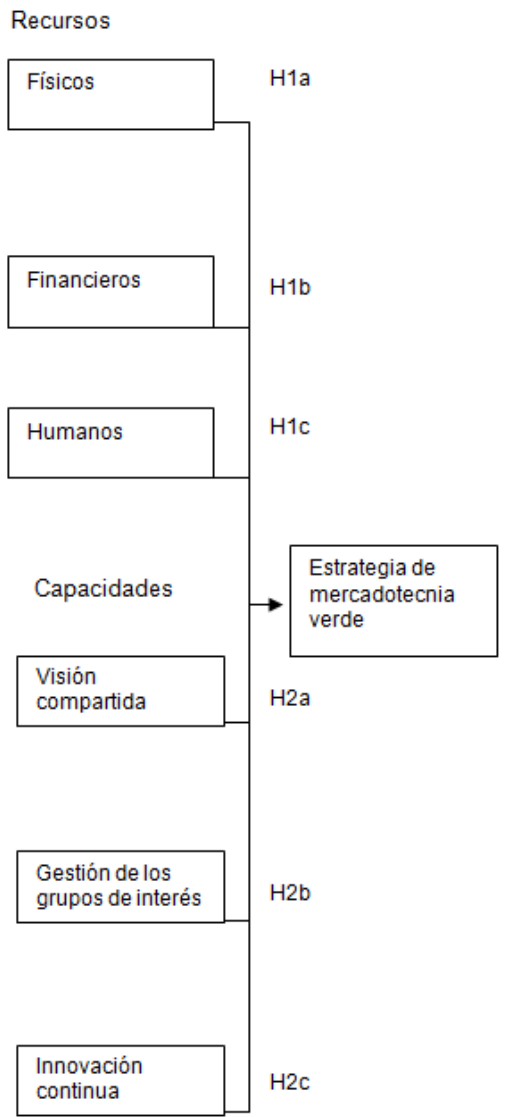

\section{Metodología}

Esta investigación se realizó en hoteles de sol y playa de Bahías de Huatulco y Puerto Escondido debido a las siguientes razones: 1) Bahías de Huatulco es un destino que posee la certificación internacional EarthCheck Gold, lo cual lo avala como una comunidad turística sustentable (Earthcheck, 2016); 2) Puerto Escondido pertenece al Programa de Turismo Sustentable de la Secretaría de Turismo del gobierno federal; el cual es una iniciativa que tiene como objetivo generar el desarrollo sustentable de la actividad turística y mejorar las condiciones en los destinos turísticos de México (SECTUR, 2013b); 3) en estos dos destinos existe un mayor número de hoteles de tres, cuatro y cinco estrellas en comparación con el resto de los destinos del estado de Oaxaca (Reyes-Santiago y Sánchez-Medina, 2016); 4) los hoteles de sol y playa tienden a ser ambientalmente más responsables y/o preocupados por llevar a cabo medidas ecoinnovadoras (Álvarez et al., 2001; García-Pozo et al., 2015). Por lo que se esperaba que los hoteles de estos dos destinos turísticos estuvieran desarrollando una EMV.

\section{Muestra}

Las hipótesis fueron probadas con datos recogidos de dueños/gerentes y/o encargados administrativos de hoteles de tres, cuatro y cinco estrellas de Bahías de Huatulco y Puerto Escondido, Oaxaca, México. Los criterios de estratificación fueron el tipo de alojamiento turístico y la categoría del hotel (número de estrellas). Con respecto al primer criterio, de los tipos de alojamiento turístico que existen en México (hoteles, apartamentos, bungalows, casas de huéspedes, suites, condominios, moteles, villas y cabañas) (SECTUR, 2013a), se eligió al hotel por ser la entidad más representativa de las empresas turísticas (Vargas et al., 2011). En cuanto al segundo criterio, se escogieron los hoteles con clasificación tres, cuatro y cinco estrellas ya que: 1) muestran homogeneidad entre ellos (García y Armas, 2007); 2) muestran ser más proactivos con el cuidado del medio ambiente (Carmona et al., 2004; Molina-Azorín, et al., 2009). La población objeto de estudio quedó conformada por los hoteles de tres, cuatro y cinco estrellas de Bahías de Huatulco y Puerto Escondido. Según los datos más recientes de la Secretaría de Turismo del gobierno federal (SECTUR, 2012), este universo estuvo compuesto por 90 hoteles. Con un nivel de confianza del 95\%, el tamaño de la muestra fue de 73 hoteles. La muestra estuvo distribuida como sigue: 37 hoteles en Bahías de Huatulco y 36 en Puerto Escondido. A continuación, se muestran las características del hotel y de la persona entrevistada (Tabla 1). 
Tabla 1 Características de la muestra.

Fuente: Elaboración propia.

\begin{tabular}{|c|c|c|c|}
\hline Unidad de análisis & Características & Categoría & Porcentaje \\
\hline \multirow{11}{*}{ Hotel } & \multirow{3}{*}{ Número de estrellas } & 3 estrellas & 42.0 \\
\hline & & 4 estrellas & 36.0 \\
\hline & & 5 estrellas & 22.0 \\
\hline & \multirow{4}{*}{ Tamaño } & $\operatorname{Micro}^{1}(0$ a 10 empleados) & 33.0 \\
\hline & & Pequeña (11 a 50 empleados) & 50.0 \\
\hline & & Mediana (51 a 100 empleados) & 14.0 \\
\hline & & Grande (más de 100 empleados) & 3.0 \\
\hline & \multirow{4}{*}{ Años de funcionamiento } & Menos de 2 años & 4.0 \\
\hline & & De 2 a 11 & 62.0 \\
\hline & & De 12 a 25 & 20.0 \\
\hline & & Más de 25 & 14.0 \\
\hline \multirow{10}{*}{ Entrevistado } & \multirow[t]{2}{*}{ Sexo } & Femenino & 70.0 \\
\hline & & Masculino & 30.0 \\
\hline & \multirow{4}{*}{ Edad } & 21 a 30 años & 36.0 \\
\hline & & 31 a 40 años & 36.0 \\
\hline & & 41 a 50 años & 12.0 \\
\hline & & Más de 50 años & 16.0 \\
\hline & \multirow{4}{*}{ Escolaridad } & Secundaria & 6.0 \\
\hline & & Bachillerato & 23.0 \\
\hline & & Licenciatura & 64.0 \\
\hline & & Posgrado & 7.0 \\
\hline
\end{tabular}

\section{Cuestionario}

El instrumento para la colecta de datos fue un cuestionario estructurado (Anexo 1), el cual fue suministrado personalmente. Se empleó una escala Likert de 5 puntos para evaluar la percepción de los entrevistados, donde 1 es "totalmente en desacuerdo" y 5 es "totalmente de acuerdo".

Como una primera fase, se llevó a cabo un análisis de contenido con nueve hoteles de tres, cuatro y cinco estrellas de los dos destinos turísticos con la finalidad de corregir y mejorar las preguntas y escalas de opinión. Esta etapa permitió mejorar la aplicabilidad del instrumento de medición en el sector hotelero. En una segunda fase, se llevó a cabo la encuesta con la muestra definitiva.

\section{Resultados y discusión}

\section{Variables medidas}

Con la finalidad de validar las escalas utilizadas en este estudio, se utilizó un análisis factorial con el método de extracción de componentes principales y rotación Varimax con normalización de Kaiser. Este método es el más usado en la literatura y tiene como finalidad reducir el número de factores que explican las variaciones en los datos, además nos permite que los factores se distingan al máximo entre sí (Hatcher, 1994).Las cargas aceptadas fueron $\geq 0.5$ (Murphy y Charles, 1998). Para el análisis de confiabilidad se utilizó el Alpha de Cronbach. 
Para medir los recursos físicos, entendidos como el equipo, maquinaria e infraestructura moderna de la empresa que facilitan la adopción de una EMV (Lucas, 2010; Russo y Fouts, 1997), se adaptaron los ítems de Leonidou et al. (2013a). Para medir los recursos financieros, entendidos como la liquidez y el poder de endeudamiento económico que permite con mayor facilidad la adopción de una EMV, se emplearon los ítems de Leonidou et al. (2013a) y Leonidou et al. (2015). Para medir los recursos humanos, entendidos como aquellas habilidades, aptitudes y conocimientos de los empleados que ayudan a la adopción de una EMV (Céspedes-Lorente y Martínez-del-Río, 2012; Fernández et al., 2003; Sarkis et al., 2010), se adaptaron los ítems de Carmona-Moreno et al. (2012) y Lucas (2010). Los recursos cargaron en los tres factores propuestos (recursos físicos, financieros y humanos) con una varianza total explicada de 74.64 y un alfa de cronbach de 0.827 (Tabla 2).
Tabla 2 Análisis factorial de la variable recursos. Fuente: Elaboración propia.

\begin{tabular}{|c|c|c|c|c|c|}
\hline \multirow{2}{*}{ Recursos } & \multicolumn{3}{|c|}{ Componente } & \multirow{2}{*}{\multicolumn{2}{|c|}{ Comunalidad }} \\
\hline & Físicos & Financieros & Humanos & & \\
\hline Su hotel posee equipo para reducir los residuos & 0.858 & 0.329 & 0.047 & 0.846 & \\
\hline Su hotel posee equipo que permita reciclar & 0.899 & 0.095 & 0.257 & 0.883 & \\
\hline $\begin{array}{l}\text { Su hotel dispone de recursos financieros necesarios para } \\
\text { dedicarlos a actividades de mercadotecnia ambiental }\end{array}$ & 0.148 & 0.675 & 0.197 & 0.516 & \\
\hline $\begin{array}{l}\text { En el hotel parte de su presupuesto se destina a } \\
\text { actividades ambientales }\end{array}$ & 0.244 & 0.807 & 0.196 & 0.748 & \\
\hline $\begin{array}{l}\text { Su hotel tiene los recursos financieros necesarios para } \\
\text { ocuparlos en actividades ambientales sin afectar el resto } \\
\text { de las operaciones del hotel }\end{array}$ & 0.103 & 0.874 & 0.106 & 0.785 & \\
\hline $\begin{array}{l}\text { Su hotel tiene la capacidad de obtener préstamos para } \\
\text { desarrollar iniciativas ambientales }\end{array}$ & 0.099 & 0.829 & 0.030 & 0.698 & \\
\hline $\begin{array}{l}\text { Sus empleados tienen las capacidades para contribuir a } \\
\text { la mejora ambiental del hotel }\end{array}$ & 0.014 & 0.319 & 0.747 & 0.659 & \\
\hline $\begin{array}{l}\text { Sus empleados tienen los conocimientos para contribuir } \\
\text { a la mejora ambiental del hotel }\end{array}$ & 0.178 & 0.127 & 0.865 & 0.796 & \\
\hline $\begin{array}{l}\text { En el hotel sus empleados tienen las habilidades para } \\
\text { contribuir a la mejora ambiental del hotel }\end{array}$ & 0.156 & 0.013 & 0.871 & 0.783 & \\
\hline Varianza explicada por dimensión & 43.38 & 18.36 & 12.90 & & \\
\hline Varianza total explicada & & & & & 74.64 \\
\hline Alfa de Cronbach por dimensión & .816 & .843 & .812 & & \\
\hline Alfa de cronbach total & & & & & 0.827 \\
\hline KMO: 0.744 & $\begin{array}{l}\text { Esfericidad } \\
\text { de Bartlett }\end{array}$ & $\begin{array}{l}\text { Chi-cuadrado } \\
294.061\end{array}$ & gl 36 & Sig. 0.000 & \\
\hline $\begin{array}{l}\text { Método de extracción: Análisis de componentes principa } \\
\text { Método de rotación: Varimax con normalización de Kais }\end{array}$ & (2) & 5 ito & & & \\
\hline
\end{tabular}


Para medir la capacidad de visión compartida, entendida como aquellos objetivos, metas, misiones y visiones comunes generadoras de la creatividad y el entusiasmo necesario para la innovación, el aprendizaje organizacional y el cambio que permiten una EMV (Hart, 1995; García-Morales y LlorensMontes, 2006; Torugsa et al., 2012), se utilizaron los ítems de Leonidou et al. (2013a).

Con la finalidad de medir la capacidad de gestión de los grupos de interés, entendida como la atención a los intereses de las autoridades, los clientes y la comunidad preocupada por los problemas ambientales a través del despliegue de una EMV (Leonidou et al., 2013a; Morgan et al., 2004; Torugsa et al., 2012), se adaptaron los ítems de Leonidou et al. (2013) y Plaza et al. (2011).

Tabla 3 Análisis factorial de la variable capacidades. Fuente: Elaboración propia.
Para medir la capacidad de innovación continua, entendida como aquella que permite a la empresa desarrollar nuevos productos y/o servicios y procesos que facilitan la adopción de una EMV (Rangone, 1999; Sharma y Vredenburg, 1998; Sharma et al., 2007), se emplearon los ítems de Christmann (1999). Las capacidades cargaron en los tres factores propuestos (visión compartida, gestión de los grupos de interés e innovación continua) con una varianza total explicada de 74.64 y un alfa de cronbach de 0.871 (Tabla 3).

\begin{tabular}{|c|c|c|c|c|}
\hline \multirow{2}{*}{ Capacidades } & \multicolumn{3}{|c|}{ Componente } & \multirow{2}{*}{ Comunalidad } \\
\hline & $\begin{array}{c}\text { Visión } \\
\text { compartida }\end{array}$ & $\begin{array}{l}\text { Gestión de los } \\
\text { grupos de interés }\end{array}$ & $\begin{array}{l}\text { Innovación } \\
\text { continua }\end{array}$ & \\
\hline $\begin{array}{l}\text { Todos sus empleados tienen una idea muy clara de los } \\
\text { objetivos ambientales de la empresa }\end{array}$ & 0.853 & 0.177 & 0.063 & 0.763 \\
\hline $\begin{array}{l}\text { Todos sus empleados hacen esfuerzos significativos } \\
\text { para alcanzar los objetivos ambientales de la empresa }\end{array}$ & 0.850 & 0.207 & 0.197 & 0.803 \\
\hline $\begin{array}{l}\text { Los gerentes y los empleados siempre se ponen de } \\
\text { acuerdo sobre el procedimiento medio ambiental para } \\
\text { la empresa }\end{array}$ & 0.816 & 0.191 & 0.088 & 0.710 \\
\hline $\begin{array}{l}\text { Los empleados a menudo ofrecen ideas valiosas para } \\
\text { mejorar las habilidades de la empresa para lograr sus } \\
\text { objetivos ambientales }\end{array}$ & 0.784 & 0.198 & 0.073 & 0.659 \\
\hline $\begin{array}{l}\text { El hotel adapta sus políticas para atender las demandas } \\
\text { de los clientes, el gobierno y la comunidad }\end{array}$ & 0.358 & 0.741 & 0.019 & 0.677 \\
\hline $\begin{array}{l}\text { El hotel da prioridad a las demandas de los clientes, el } \\
\text { gobierno y la comunidad }\end{array}$ & 0.375 & 0.792 & 0.056 & 0.771 \\
\hline $\begin{array}{l}\text { El hotel compromete tiempo y recursos para adaptarse } \\
\text { a las demandas de los clientes, el gobierno y la } \\
\text { comunidad }\end{array}$ & 0.075 & 0.913 & 0.130 & 0.856 \\
\hline $\begin{array}{l}\text { Los directivos del hotel discuten acerca de las } \\
\text { demandas de los clientes, el gobierno y la comunidad. }\end{array}$ & 0.267 & 0.605 & 0.204 & 0.479 \\
\hline $\begin{array}{l}\text { En el hotel es importante conocer las demandas de los } \\
\text { clientes, el gobierno y la comunidad }\end{array}$ & 0.035 & 0.761 & 0.172 & 0.610 \\
\hline El hotel utiliza nuevas tecnologías en sus procesos & 0.041 & 0.053 & 0.857 & 0.738 \\
\hline El hotel realiza cambios en sus procesos & 0.279 & 0.142 & 0.741 & 0.647 \\
\hline $\begin{array}{l}\text { El hotel desarrolla productos y servicios nuevos o } \\
\text { mejorados }\end{array}$ & 0.030 & 0.202 & 0.815 & 0.707 \\
\hline Varianza explicada por dimensión & 14.61 & 42.24 & 13.32 & \\
\hline Varianza total explicada & & & & 70.17 \\
\hline Alfa de Cronbach por dimensión & 0.877 & 0.857 & 0.765 & \\
\hline Alfa de cronbach total & & & & 0.871 \\
\hline KMO: 0.799 & $\begin{array}{l}\text { Esfericidad de } \\
\text { Bartlett }\end{array}$ & $\begin{array}{c}\text { Chi-cuadrado } \\
459.455\end{array}$ & $\mathrm{gl} 66$ & Sig. 0.000 \\
\hline $\begin{array}{l}\text { Método de extracción: Análisis de componentes princip } \\
\text { Método de rotación: Varimax con normalización de Kai }\end{array}$ & rotación ha co & lo en 5 iteraciones. & & \\
\hline
\end{tabular}


Para medir la EMV, entendida como aquellas actividades de la mezcla de mercadotecnia (producto/servicio precio, plaza y promoción) que el hotel realiza para satisfacer las expectativas de los clientes al mismo tiempo que minimiza su impacto al medio ambiente (Fraj et al., 2011; Nandini y Deshpande, 2011; Taghian et al., 2016), se adaptaron los ítems de Leonidou et al. (2013a). Como se observa en la tabla 4, la EMV sólo cargó en dos (producto/servicio, promoción) de los cuatro factores propuestos (producto/servicio precio, plaza y promoción) con una varianza total explicada de 64.36 y un alfa de cronbach de 0.687 .
Tabla 4 Análisis factorial de la variable estrategia de mercadotecnia verde. Fuente: Elaboración propia.

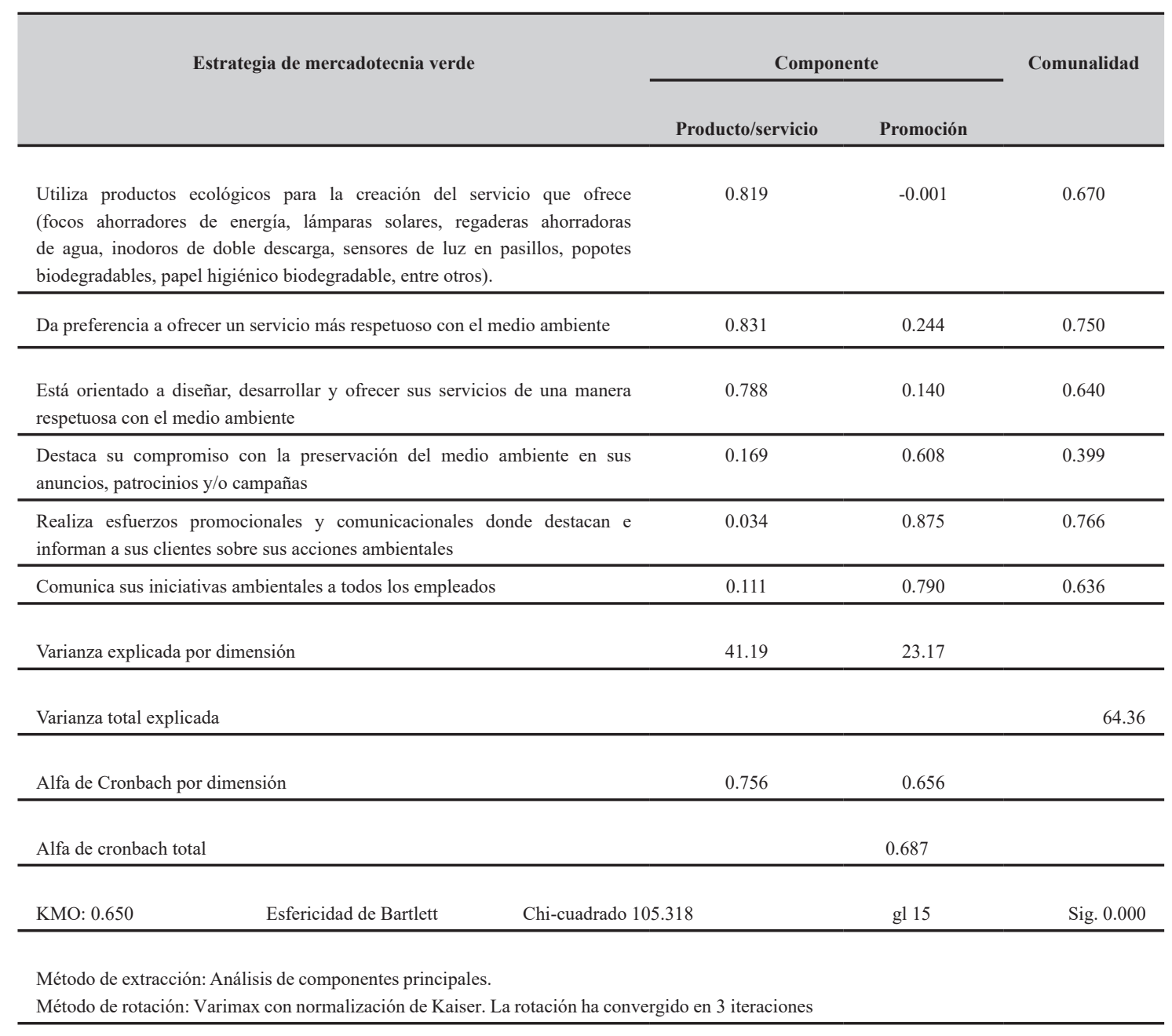

Por otra parte, se halló que los recursos financieros tienen una relación positiva $\mathrm{y}$ significativa de $\mathrm{r}=.245$ con una $\mathrm{p} \leq .05$ con la EMV. Con base en lo anterior, se acepta la $\mathrm{H} 1 \mathrm{~b}$. Este resultado concuerda con la literatura al indicar que los recursos financieros son fundamentales para lograr la permanencia y el éxito de la EMV (Chan, 2005; Leonidou et al., 2013a; Shah, 2011). Este resultado representa una buena señal para la gestión ambiental en la hotelería, porque en algunas ocasiones, aunque los hoteles reconocen sus impactos ambientales y desean realizar prácticas ambientales, no siempre cuentan con los recursos necesarios para realizarla (López-Moreda y VargasMartínez, 2013). En los hoteles de la muestra, el poseer recursos financieros permite destinar un presupuesto a actividades ambientales. En los hoteles, existen muchas fuentes de contaminación porque el impacto ambiental está fragmentado en muchas actividades; de ahí la importancia de poseer recursos financieros para atender a cada una de estas fuentes contaminantes. 
Además, se encontró que los recursos humanos tienen una relación positiva y significativa de $\mathrm{r}=.432$ con una $\mathrm{p} \leq .01$ con la EMV. Esta información apoya la H1c. Este resultado coincide con la literatura al indicar que los recursos humanos son fundamentales para las empresas que buscan adoptar una estrategia más responsable con el entorno natural (Aragón y Sharma, 2003; Fernández et al., 2003; Vidal et al., 2012). Debido a que en un hotel el impacto ambiental es fragmentado, la forma de disminuirlo es a través de actividades de una
EMV en cada una de estas fuentes de contaminación, y es ahí precisamente en donde las habilidades y conocimientos ambientales de los empleados son relevantes porque son quienes se encargan de cada una de estas acciones.

Con base en estos resultados, es posible notar la importancia de que los hoteles adquieran y mantengan recursos tangibles e intangibles adecuados para desplegar programas de una estrategia más responsable con el medio ambiente (Leonidou et al. 2013a).
Tabla 5 Correlación bivariada de Pearson entre las variables de estudio. Fuente: $\quad$ Elaboración propia.

\begin{tabular}{|c|c|c|c|c|c|c|c|c|c|c|}
\hline Variable & Media & $\begin{array}{c}\text { Desviación } \\
\text { estándar }\end{array}$ & $\mathbf{N}$ & 1 & 2 & 3 & 4 & 5 & 6 & 7 \\
\hline $\begin{array}{l}1 \text { Recursos } \\
\text { físicos }\end{array}$ & 2.4658 & 0.80073 & 73 & 1 & $0.341^{* *}$ & $0.365^{* *}$ & $0.305^{* *}$ & 0.157 & 0.183 & 0.135 \\
\hline $\begin{array}{l}2 \text { Recursos } \\
\text { financieros }\end{array}$ & 2.5342 & 0.60282 & 73 & & 1 & $0.289 *$ & 0.142 & $0.263^{*}$ & $0.415^{* *}$ & $0.245^{* *}$ \\
\hline $\begin{array}{l}3 \text { Recursos } \\
\text { humanos }\end{array}$ & 2.6575 & 0.50605 & 73 & & & 1 & $0.395^{* *}$ & $0.241^{*}$ & $0.324 * *$ & $0.432 * *$ \\
\hline $\begin{array}{l}4 \text { Capacidad } \\
\text { de visión } \\
\text { compartida }\end{array}$ & 2.6438 & 0.63179 & 73 & & & & 1 & $0.333^{* *}$ & $0.249^{*}$ & $0.334^{* *}$ \\
\hline $\begin{array}{l}5 \text { Capacidad de } \\
\text { gestión de los } \\
\text { grupos de interés }\end{array}$ & 2.7945 & 0.43966 & 73 & & & & & 1 & 0.191 & $0.313^{* *}$ \\
\hline $\begin{array}{l}6 \text { Capacidad } \\
\text { de innovación } \\
\text { continua }\end{array}$ & 2.8219 & 0.38523 & 73 & & & & & & 1 & 0.149 \\
\hline 7 EMV & 2.7534 & 0.43400 & 73 & & & & & & & 1 \\
\hline $\begin{array}{l}{ }^{*} \mathrm{p}<0.05 \\
* * \mathrm{p}<0.01\end{array}$ & & & & & & & & & & \\
\hline
\end{tabular}

Asimismo, se halló que la capacidad de visión compartida tiene una relación positiva y significativa de $\mathrm{r}=.334$ con una $\mathrm{p} \leq .01$ con la EMV. Esta información permite aceptar la $\mathrm{H} 2 \mathrm{a}$. Este resultado concuerda con lo encontrado en la literatura al señalar que las empresas que poseen una visión compartida tienen más probabilidades de adoptar una estrategia más amigable con el medio ambiente (Chan, 2005; Hart, 1995; Leonidou et al., 2013a). El hecho de que todos los empleados compartan la visión ambiental de la empresa favorece el desarrollo de una EMV porque, en un hotel las prácticas ambientales predominantes son aquellas en donde participan todos los empleados, es decir, aquellas que se pueden realizar durante sus procesos normales de operación, y aquellas guiadas para reducir costos (Garay y Font, 2012).

También, se encontró que la capacidad de gestión de los grupos de interés tiene una relación positiva y significativa de $r=.313$ con una $p \leq .01$ con la EMV. Estos resultados permiten aceptar la $\mathrm{H} 2 \mathrm{~b}$. Lo anterior coincide con la literatura académica, la cual indica que la gestión de los grupos de interés permite el desarrollo de iniciativas ambientales (Álvarez et al., 2001; Bianchi y Nochi, 1998; Plaza et al., 2011; Hart, 1995; Torugsa et al., 2012). En la muestra, el atender las demandas ambientales de los clientes, gobierno y comunidad es un factor que conduce a una estrategia de mercadotecnia responsable con el medio ambiente. Este resultado podría deberse a que los hoteles perciben que la presión ambiental que ejercen estos grupos de interés es significativa y por ello, es necesario atenderla. Como cada vez existe mayor conciencia ambiental entre los grupos de interés que ejercen presión ambiental en el sector turístico, para los hoteles de la muestra resulta importante gestionar las solicitudes en el tema ambiental que realizan dichos grupos.

Por último, los resultados muestran que la capacidad de innovación continua tiene una relación positiva $y$ no significativa de $\mathrm{r}=.149$ con una $\mathrm{p}>.05$ con la EMV. Estos resultados no permiten aceptar la H2c. Este resultado no coincide con la literatura académica, la cual indica que la capacidad de innovación continua en los procesos de la empresa ayuda a desarrollar una EMV (Christmann, 2000; González-Benito y GonzálezBenito, 2005; Sharma y Vredenburg, 1998). Los hoteles ofrecen numerosos ejemplos de innovación, los cuales se pueden hallar tanto en la gestión de recursos humanos, en la cadena de suministro como en la gestión de la rentabilidad (Hertog et al., 2011). Sin embargo, en la muestra de hoteles se percibe que la capacidad de 
innovación continua no representa un factor que les permita desarrollar una EMV. La razón podría radicar en que la mayoría de los hoteles encuestados declaró no realizar innovaciones en sus productos/servicios y procesos. Podría ser que, para estos hoteles, las innovaciones son vistas como un elemento que se desarrolla para reaccionar ante una contingencia, pero no como un factor que les permita obtener éxito empresarial.

\section{Conclusiones}

En la discusión actual académica y de gestión de las empresas sobre transitar de una estrategia de mercadotecnia tradicional a una EMV, este estudio evidencia que los recursos y capacidades internos conducen a adoptar una EMV en el sector servicios, particularmente en el sector hotelero de sol y playa de Bahías de Huatulco y Puerto Escondido, Oaxaca. Se encontró que los recursos financieros y humanos y las capacidades de visión compartida y de gestión de los grupos de interés contribuyen positivamente a la adopción de una EMV en la muestra de hoteles estudiados.

Con lo anterior, esta investigación contribuye a la TBR al mostrar la relación positiva de los recursos y capacidades en la adopción de una EMV en el sector hotelero. Asimismo, este estudio contribuye a llenar el vacío en la literatura académica al examinar una EMV al interior de las empresas hoteleras, por lo cual, también se hace un aporte a la literatura académica sobre mercadotecnia verde.

La adopción de una EMV en hoteles de sol y playa reviste de especial importancia debido a que, sus clientes buscan realizar actividades que implican el disfrute de los recursos naturales (Álvarez et al., 1999). Por lo que, se podría esperar que los hoteles de este tipo tengan una mayor preocupación ambiental que el resto de las categorías (negocios, de salud, entre otras) y, por lo tanto, desarrollen una EMV. Por lo que, esta investigación tiene implicaciones para la gestión de la empresa hotelera de sol y playa, ya que provee conocimiento sobre los recursos y capacidades tangibles e intangibles que influyen positivamente para adoptar una EMV.

Finalmente está investigación es de utilidad para las diferentes asociaciones de la actividad turística como la Asociación Mexicana de Hoteles y Moteles y las secretarías de turismo porque da evidencia de que los hoteles de 3, 4 y 5 estrellas de Bahías de Huatulco y Puerto Escondido, Oaxaca, usan sus recursos físicos, financieros y humanos, y las capacidades de visión compartida y gestión de los grupos de interés para adoptar una EMV. Estos hoteles han encontrado beneficios económicos como el maximizar sus ganancias a partir de la implementación de una EMV.

Asimismo este estudio tiene implicaciones para el gobierno ya que deja ver iniciativas que puede implementar como apoyo con créditos o financiamiento a hoteles de menor tamaño y clasificación con la finalidad de que puedan adquirir recursos físicos enfocados en el cuidado del medio ambiente, pues se observó que existen hoteles que por limitaciones financieras no pueden adquirir equipo o tecnología limpia a diferencia de hoteles grandes de cinco estrellas, quienes tienen mayor liquidez financiera.

También se hace evidente la necesidad de ofrecer información y capacitación a los recursos humanos en los hoteles sobre la importancia de implementar prácticas ambientales que les conduzcan en la implementación de una EMV.

\section{Referencias bibliográficas}

1. Álvarez, M.J., DE BURGOS, J., y CÉSPEDES, J.J. (1999). «Estrategia medioambiental, contexto organizativo y rendimiento: los establecimientos hoteleros españoles». Serie de Economía de la Empresa 05, Departamento de Economía de la Empresa, Universidad Carlos III de Madrid, documento de trabajo, pp.1-28.

2. ÁlVAREZ, M.J., DE BURGOS, J., y CÉSPEDES, J.J. (2001). «An analysis of environmental management organizational context and performance of Spanish hotels», Omega The International Journal of Management Science, 29, pp. 457-471.

3. ARAGÓN, J., y SHARMA, S. (2003). «A contingent resource-based view of proactive corporate environmental strategy». Academy of management review, pp. 71-88.

4. ARAGÓN-CORREA, J.A., GARCÍA-MORALES, V.J., y HURTADO-TORRES, N.E. (2005). «Un modelo explicativo de las estrategias medioambientales avanzadas para pequeñas y medianas empresas y su influencia en los resultados». Cuadernos de Economía y Dirección de la Empresa, 25, pp. 29-51.

5. ARMAS-CRUZ, Y. (2011). Determinants of environmental management and implications for firms' economic performance». Journal of Environmental Planning and Management, 54(8), pp. 1077-1106. doi: $10.1080 / 09640568.2010 .549401$

6. BARNEY, J. (1986). «Organizational culture: can it be a source of SCA?». Academy of Management Review, 11 (3), pp. 656-665.

7. BARNEY, J. (1991). «Firm resources and sustained competitive advantage». Journal of Management, 17(1), pp. 99-120. 
8. BIANCHI, R., y NOCI, G. (1998). "Greening SME's competitiveness». Small business economics, 11, pp. 269-281.

9. CARMONA, M., CÉSPEDES, L., y BURGOS, J. (2004). «Environmental strategies in Spanish hotels: contextual factors and performance». The Service Industries Journal, 24(3), pp. 101-130.

10. CARMONA-MORENO, E., CÉSPEDES-LORENTE, J., y MARTÍNEZ-DEL-RÍO, J. (2012). «Environmental human resource management and competitive advantage». Management Research, 10(2), pp. 125-142.

11. CEA D' ANCONA, M. (2001). Metodología Cuantitativa: Estrategias y técnicas de investigación social. Madrid: Ed. Síntesis.

12. CÉSPEDES, J., y DE BURGOS, J. (2004). «Un análisis de las dimensiones de la gestión ambiental en los servicios hote $\neg$ leros». Revista de Ingeniería de Organización, 30, pp. 119-237.

13. CHAN, R. (2005). «Does the natural-resource-based view of the firm apply in an emerging economy? A survey of foreign invested enterprises in China». Journal of management studies, 42(3), pp. 626-672.

14. CHAN, E. (2013). «Managing green marketing: Hong Kong hotel managers' perspective». International Journal of Hospitality Management, 34, pp. 442-461.

15. CHAN, E. (2014). «Green marketing hotel customers' perspective». Journal of Travel \& Tourism Marketing, 31(8), pp. 915-936.

16. CHRISTMANN, P. (2000). «Effects of 'best practices' of environmental management on cost advantage: The role of complementary assets». Academy of Management Journal, pp. 663-680.

17. CONNER, K. R. (1991). "A historical comparison of resource-based theory and five schools of thought within industrial organization economics: do we have a new theory of the firm?». Journal of Management, 17 (1), pp. 121-154.

18. CRUZ, D., JACOBO, C.A., OCHOA, S., y LEYVA, B. (2014). «Desempeño sustentable en establecimientos de hospedaje de ciudad Obregón, Sonora». Revista Interamericana de Ambiente y Turismo, 10 (1), pp. 5566.
19. CRUZ-BAUTISTA, M. (2013). «Relación entre los intereses ambientales de stakeholders y los sistemas de gestión ambiental en el sector hotelero oaxaqueño». México. Ed. Instituto Politécnico Nacional.

20. DAY, G. (1994). «The capabilities of market-driven organizations». Journal of Marketing, 58(4), pp. 37-52.

21. DE YTA-CASTILLO, D., y SÁNCHEZ-MEDINA, P. S. (2017). «Estrategias ambientales y desempeño empresarial de hoteles de México».Temas de Ciencia y Tecnología, 21(63), pp. 5-21.

22. DE YTA-CASTILLO, D., SÁNCHEZ-MEDINA, P. S., y RAMÍREZ, J.A. (2018). «Compromiso ambiental, desempeño ambiental y desempeño: un estudio exploratorio en hoteles de tres, cuatro y cinco estrellas de cinco entidades de México». CIENCIA-ergo sum, 26(2), pp. e51.

23. DIERICKX, I., y COOL, K. (1989). «Asset stock accumulation and sustainability of competitive advantage». Management Science, 35(12), pp. 15041511 .

24. Earthcheck (2016). Huatulco, 2do. Destino turístico a nivel mundial con la certificación EarthCheck Platinum. Fecha de consulta: 23 de enero de 2020. Obtenido de: https://earthcheck.org/news/2016/august/huatulco-2dodestino-tur $\% \mathrm{C} 3 \%$ ADstico-a-nivel-mundial-con-lacertificaci $\% \mathrm{C} 3 \% \mathrm{~B} 3$ n-earthcheck-platinum/

25. EISENHARDT, K., y MARTIN, J. (2000). «Dynamic capabilities: what are they?». Strategic Management Journal, 21(10-11), pp. 1105-1121.

26. EL DIEF, M., y FONT, X. (2010). «The determinants of hotels' marketing managers' green marketing behaviour». Journal of Sustainable Tourism, 18(2), pp. 157-174.

27. FERNÁNDEZ, E., JUNQUERA, B., y ORDIZ, M. (2003). «Organizational culture and human resource in the environmental issue: a review of the literature». The International Journal of Human Resource Management, 14(4), pp. 634-656.

28. FERNÁNDEZ, M.T., y CUADRADO, R. (2011). «La responsabilidad social empresarial en el sector hotelero: revisión de la literatura científica». Cuadernos de Turismo, 28, pp. 47-57. 
29. FONG, C. (2011). «La teoría de recursos y capacidades: un enfoque contemporáneo en la gestión empresarial». Carta Económica Regional, 61, pp. 27-31.

30. FRAJ, E., MARTÍNEZ, E., y MATUTE, J. (2011). «Green marketing strategy and the firm's performance: the moderating role of environmental culture». Journal of Strategic Marketing, 19(4), pp. 339-355.

31. FRAJ, E., MATUTE, J., y MELERO, I. (2015). «Environmental strategies and organizational competitiveness in the hotel industry: The role of learning and innovation as determinants of environmental success». Tourism Management, 46, pp. 30-42.

32. GARAY, L., y FONT, X. (2012). «Doing good to do well? Corporate social responsibility reasons, practices, and impacts in small and medium accommodation enterprises». International Journal of Hospitality Management, 31, pp. 329-337.

33. GARCÍA-MORALES, V., y LLORENS-MONTES, F. (2006). «Antecedents and consequences of organizational innovation and organizational learning in entrepreneurship». Industrial Management \& Data Systems, 106(1), pp. 21-42.

34. GARCÍA-POZO, A., SÁNCHEZ-OLLERO, J., y MARCHANTE-LARA, M. (2015). «Ecoinnovation and management: an empirical analysis of environmental good practices and labour productivity in the spanish hotel industry». Innovation: Organization \& Management, 17(1), pp. 58-68.

35. GRANT, R. (1991). "The resource-based theory of competitive advantage: implications for strategy formulation». California Management Review, 33(3), pp. 114-135.

36. GAVRONSKI, L., KLASSEN, D., VACHON, S., y MACHADO, L. (2011). «A resource-based view of green supply management». Transportation Research Part E: Logistics and Transportation Review, 47(6), pp. 872-885.

37. GONZÁLEZ-BENITO, J., y GONZÁLEZ-BENITO, O. (2005). «Environmental proactivity and business performance: an empirical analysis». The International Journal of Management Science, 33(1), pp. 1-15.

38. HART, S. (1995). «A natural-resource-based view of the firm». Academy of Management Review, 20(4), pp. 986104.
39. HART, S.L., y MILSTEIN, M.B. (2003). «Creating sustainable value». Academy of Management Executive, 17 (2), pp. 56-67.

40. HART, S.L., y DOWELL, G. (2011). «A naturalresource-based view of the firm: fifteen years after». Journal of Management, 37 (5), pp. 1464-1479.

41. HATCHER, L. (1994). A step by step approach to using the SAS system for factor analysis and structural equation modeling. Cary, NC, USA: SAS Institute Inc.

42. HertoG, P., DEN GAllouj, F., y SEGERS, J., 2011. "Measuring innovation in a low-tech service industry: the case of the Dutch hospitality industry». The Service Industries Journal, 31 (9), pp. 1429-1449. Instituto Nacional de Estadística y Geografía (INEGI). (2011). Micro, pequeña, mediana y gran empresa. Censos Económicos 2009. México.

43. KASIM, A., GURSOY, D., OKUMUS, F., y WONG, A. (2014). «The importance of water management in hotels: a framework for sustainability through innovation». Journal of Sustainability Tourism, 22(7), pp.1090-1107.

44. KIRK, D. (1995). «Environmental management in hotels». International Journal of Contemporary Hospitality Management, 7(6), pp. 3-8.

45. LEONIDOU, C., y LEONIDOU, L. (2011). «Research into environmental marketing/management: a bibliographic analysis». European Journal of Marketing, 45(1/2), pp. 68-103.

46. LEONIDOU, L., LEONIDOU, C., FOTIADIS, T., y ZERITI, A. (2013a). «Resources and capabilities as drivers of hotel environmental marketing strategy: Implications for competitive advantage and performance». Tourism Management, 35, pp. 94-110.

47. LEONIDOU, C., KATSIKEAS, C., y MORGAN, N. (2013b). «"Greening" the marketing mix: do firms it and does it pay off? ». Journal of the Academy of Marketing Science, 41(2), pp. 1-22.

48. LEONIDOU, L., FOTIADIS, T., CHRISTODOULIDES, P., SPRYROPOULOU, S., y KATSIKEAS, C. (2015). «Environmentally export business strategy: its determinants and effects on competitive advantage and performance». International Business Review, 24(5), pp. 798-811. 
49. LÓPEZ-GAMERO, M. D., MOLINA-AZORÍN, J.F., y CLAVER-CORTÉS, E. (2009). «The whole relationship between environmental variables and firm performance: competitive advantage and firm resources as mediator variables». Journal of Environmental Management, 90(10), pp. 3110-3121.

50. LÓPEZ-GAMERO, M., CLAVER-CORTÉS, E., y MOLINA-AZORÍN, F. (2011a). «Environmental perception, management and competitive opportunity in Spanish hotels». Cornell Hospitality Quarterly, 52(4), pp. $480-500$.

51. LÓPEZ-MOREDA, L.J. y VARGAS-MARTÍNEZ, E.E. (2013). «Evaluación preliminar de la gestión ambiental en hoteles del destino turístico de Varadero, Cuba». Teoría y Praxis, 13, 133-150.

52. LUCAS, M. (2010). «Understanding environmental management practices: integrating views from strategic management and ecological economics». Business Strategy and the Environment, 19(8), pp. 543-556.

53. MATUTE, J., y MELERO, I. (2015). «Environmental strategies and organizational competitiveness in the hotel industry: The role of learning and innovation as determinants of environmental success». Tourism Management, 46 pp. 30-42.

54. MEDINA-SERRANO, R., GONZÁLEZ-RAMÍREZ, M., GASCÓ, J.L., y LLOPIS, J. (2019). «Sustainable supplier evaluation practices across the supply chain». Dirección y Organización, 69, pp. 13-26.

55. MENGUC, B., Auh, S. y Ozzane, L. (2010). «The interactive effect of internal and external factors on a proactive environmental strategy and its influence on a firm's performance», Journal of Business Ethics, 94(2), pp. $279-298$.

56. MOLINA-AZORÍN, J. F., CLAVER-CORTÉS, E., PEREIRA-MOLINER, J., y TARÍ, J. J. (2009). «Environmental practices and firm performance: An empirical analysis in the Spanish hotel industry». Journal of Cleaner Production, 17(5), pp. 516-524.

57. MURPHY, K. R., y CHARLES, O. D. (1998). Psychological Testing: Principles and Applications. Upper Saddle River, N.J. Ed. Prentice-Hall.

58. NANDINI, M., y DESHPANDE, M. (2011). «A conceptual framework on green marketing -a tool for sustainable development». International Journal of Sales and Marketing Management, 1(1), pp. 1-196.
59. PAULRAJ, A. (2011). «Understanding the relationship between internal resources and capabilities, sustainable supply management and organizational sustainability». Journal of Supply Chain Management, 47(1), pp. 19-37.

60. PLAZA, J., DE BURGOS, J., y BELMONTE, L. (2011). «Grupos de interés, gestión ambiental y resultado empresarial: una propuesta integradora». Cuadernos de Economía y Dirección de la Empresa, 14(3), pp.151161.

61. PRAKASH, A. (2002). «Green marketing, public policy and managerial strategies». Business Strategy and the Environment, 11, pp. 285-297.

62. RADWAN, H., JONES, E., y MINOLI, D. (2012). «Solid waste management in small hotels: a comparison of green and non-green small hotels in Wales». Journal of Sustainable Tourism, 20(4), pp. 533-550.

63. RANGONE, A. (1999). «A resource-based aproach to strategy analysis in small-medium sized enterprises». Small Business Economics, 12, pp. 233-248.

64. REED, R., y DEFILLIPPI, R.J. (1990). «Causal ambiguity, barriers to imitation, and sustainable competitive advantage». The Academy of Management Review, 15 (1), pp. 88-102.

65. REYES-SANTIAGO, M., y SÁNCHEZ-MEDINA, P. S. (2016). «Eco-innovación en empresas hoteleras de Oaxaca, México». Salud y Administración, 3(8), pp. 2737.

66. REYES-SANTIAGO, M., SÁNCHEZ-MEDINA, P. S., y DÍAZ-PICHARDO, R. (2017). «Eco-innovation and organizational culture in the hotel industry». International Journal of Hospitality Management, 65, pp. 71-80.

67. REYES-SANTIAGO, M., SÁNCHEZ-MEDINA, P. S., y DÍAZ-PICHARDO, R. (2019). «The influence of environmental dynamic capabilities on organizational and environmental performance of hotels: evidence from Mexico». Journal of Cleaner Production, 227, pp. 414-423.

68. RIVERA, J. (2002). «Assessing a voluntary environmental initiative in the developing world: The Costa Rican Certification for Sustainable Tourism». Policy Sciences, 35, pp. 333-360. 
69. RUSSO, M., y FOUTS, P. (1997). «A resource-based perspective on corporate enviromental performance and profitability». Academy of Management Journal, 40(3), pp. 534-559.

70. SÁNCHEZ-MEDINA, P. S., DÍAZ-PICHARDO, R., y CRUZ-BAUTISTA, M. (2016). «Stakeholder influence on the imple $\neg$ mentation of environmental management practices in the hotel industry». International Journal of Tourism Research, 18(4), pp. 387-398.

71. SARKIS, J., GONZÁLEZ-TORRE, P., y ADENSODÍAZ, B. (2010). «Stakeholder pressure and the adoption of environmental practices: the mediating effect of training». Journal of Operations Management, 28(3), pp. 163-176.

72. SECRETARÍA DE TURISMO (SECTUR) (2012). «Compendio Estadístico del Turismo en México 2012». México. Ed. DATATUR, SECTUR.

73. SECRETARÍA DE TURISMO (SECTUR) (2013a). «Acerca de nosotros: Compendio Estadístico del Turismo en México». Fecha de consulta: 10 de diciembre de 2019.

74. SECRETARÍA DE TURISMO (SECTUR) (2013b). «Comité de información: Agenda 21 para el Turismo Mexicano». Puerto Escondido, Oaxaca: SECTUR.

75. SECRETARÍA DE TURISMO (SECTUR) (2016). Indicadores de la actividad turística Enero-Diciembre del 2016. México. Ed. SECTUR.

76. SHAH, K. (2011). «Strategic organizational drivers of corporate environmental responsibility in the Caribbean hotel industry». Policy Sciences, 44(4), pp. 321-344.

77. SHARMA, S., y VREDENBURG, H. (1998). «Proactive corporate environmental strategy and development of competitively valuable organizational capabilities». Strategic Management Journal, 19(8), pp. 729-753.

78. SHARMA, S., ARAGÓN-CORREA, J., y RUEDAMANZANARES, A. (2007). «The contingent influence or organizational capabilities on proactive environmental strategy in the service sector: An analysis of North American and European ski resorts». Canadian Journal of Administrative Science, 24(4), pp. 268-283.

79. SURROCA, J., TRIBÓ, J., y WADDOCK, S. (2010). «Corporate responsibility and financial performance: the role of intangible resources». Strategic Management Journal, 31(5), pp. 463-490.
80. TAGHIAN, M., POLONSKY, M., y D'SOUZA, C. (2016). «Green marketing strategies». In An Integrated Approach to Environmental Management. Edited by SARKAR, D., DATTA, R., MUKHERJEE, A. First Edition, John Wiley \& Sons, pp. 231-256.

81. TENG, C. C., HORNG, J. S., HU, M. L., CHIEN, L. H., y SHEN, Y. C. (2012). «Developing energy conservation and carbon reduction indicators for the hotel industry in Taiwan». International Journal of Hospitality Man $\neg$ agement, 31(1), pp. 199-208.

82. TIWARI, S., TRIPATHI, D.M., SRIVASTAVA, U., y YADAV, P.K. (2011). "Green marketing-emerging dimensions». Journal of Business Excellence, 2(1), pp. 18-23.

83. TORUGSA, N., O'DONOHUE, W., y HECKER, R. (2012). «Capabilities, proactive CSR and financial performance in SMEs: empirical evidence from an Australian manufacturing industry sector». Journal of Business Ethics, 109(4), pp. 483-500.

84. VARGAS, E., ZIZUMBO, L., VIESCA, F., y SERRANO, R. (2011). «Gestión ambiental en el sector turístico mexicano. Efectos de la regulación en el desempeño hotelero». Cuadernos de Administración, 24(42), pp. 183-204.

85. VARGAS, E. E. (2015). Responsabilidad social empresarial y gestión ambiental en el sector hotelero. Toluca. Ed. Universidad Autónoma del Estado de México.

86. VELÁZQUEZ-CASTRO, J.A., VARGAS-MARTÍNEZ, E.E., OLÍVER-ESPINOZA, R., y CRUZ-JIMÉNEZ, G. (2016). «Elementos determinantes de la ecoinnovación en hotelería de Huatulco, México». Revista Venezolana de Gerencia, 21(74), pp. 242-256.

87. VIDAL, M., CORDÓN, E., DELGADO, J., y GÓMEZ, S. (2012). «Proactividad medioambiental en el sector hotelero: influencia de los procesos de aprendizaje». Tribuna de Economía, pp. 133-151.

88. YIN, H., y SCHMEIDLER, P.J. (2009). «Why do standardized ISO 14001 environmental management systems lead to heterogeneous environmental outcomes?». Business Strategy and the Environment, 18(7), pp. 469-486. 
Anexo 1 Cuestionario empleado en el estudio.

Nota: Las preguntas en letra negrita son las que resultaron válidas y confiables.

\begin{tabular}{|c|c|c|c|c|}
\hline Autor & Variable & Dimensiones & Preguntas & Escala \\
\hline \multirow{4}{*}{ Leonidou et al. (2013a) } & \multirow{16}{*}{ Recursos } & \multirow{4}{*}{ Físicos } & $\begin{array}{l}\text { Su hotel posee equipo para reducir los residuos } \\
\text { (separadores, trituradoras, etc.) }\end{array}$ & \multirow{16}{*}{$\begin{array}{l}\text { Tipo Likert de } 5 \text { puntos, } \\
\text { donde } 1 \text { es "totalmente en } \\
\text { desacuerdo" y } 5 \text { "totalmente } \\
\text { de acuerdo. }\end{array}$} \\
\hline & & & $\begin{array}{l}\text { Su hotel posee equipo que permita reciclar (planta de } \\
\text { tratamiento de aguas residuales) }\end{array}$ & \\
\hline & & & $\begin{array}{l}\text { Su hotel utiliza equipos que ahorran agua (grifos con sistema } \\
\text { de sensores, uso de regaderas de bajo flujo, inodoros de doble } \\
\text { descarga) }\end{array}$ & \\
\hline & & & $\begin{array}{l}\text { Su hotel utiliza equipos que ahorran energía (equipo de cómputo, } \\
\text { sensores de movimiento ahorradores de luz, aires acondicionados, } \\
\text { transporte ahorrador de energía, tarjetas electrónicas de entrada } \\
\text { a la habitación, etc.) }\end{array}$ & \\
\hline \multirow{5}{*}{$\begin{array}{l}\text { Leonidou et al. (2013a); } \\
\text { Leonidou et al. (2015). }\end{array}$} & & \multirow{5}{*}{ Financieros } & $\begin{array}{l}\text { Su hotel dispone de recursos financieros necesarios } \\
\text { para dedicarlos a actividades de mercadotecnia ambiental }\end{array}$ & \\
\hline & & & $\begin{array}{l}\text { En el hotel parte de su presupuesto se destina a } \\
\text { actividades ambientales }\end{array}$ & \\
\hline & & & $\begin{array}{l}\text { Su hotel tiene los recursos financieros necesarios para } \\
\text { ocuparlos en actividades ambientales sin afectar el resto de } \\
\text { las operaciones del hotel }\end{array}$ & \\
\hline & & & $\begin{array}{l}\text { Su hotel tiene la capacidad de obtener préstamos para } \\
\text { desarrollar iniciativas ambientales }\end{array}$ & \\
\hline & & & $\begin{array}{l}\text { Su hotel puede obtener rápidamente préstamos para las } \\
\text { actividades de mercadotecnia ambiental }\end{array}$ & \\
\hline \multirow{7}{*}{$\begin{array}{l}\text { Carmona-Moreno et al. (2012); } \\
\text { Lucas (2010) }\end{array}$} & & \multirow{7}{*}{ Humanos } & $\begin{array}{l}\text { Sus empleados tienen las capacidades para contribuir a la } \\
\text { mejora ambiental del hotel }\end{array}$ & \\
\hline & & & $\begin{array}{l}\text { Sus empleados tienen los conocimientos para contribuir } \\
\text { a la mejora ambiental del hotel }\end{array}$ & \\
\hline & & & $\begin{array}{l}\text { Sus empleados tienen las habilidades para contribuir a la } \\
\text { mejora ambiental del hotel }\end{array}$ & \\
\hline & & & Sus empleados están capacitados en el tema ambiental & \\
\hline & & & Sus empleados están educados en el tema ambiental & \\
\hline & & & $\begin{array}{l}\text { Sus empleados tienen la iniciativa de ahorrar agua, energía } \\
\text { eléctrica, reciclar, disminuir el uso de insumos, entre otras } \\
\text { actividades que contribuyen a la mejora ambiental del hotel }\end{array}$ & \\
\hline & & & $\begin{array}{l}\text { Sus empleados buscan ser líderes en el tema de las iniciativas } \\
\text { ambientales }\end{array}$ & \\
\hline
\end{tabular}




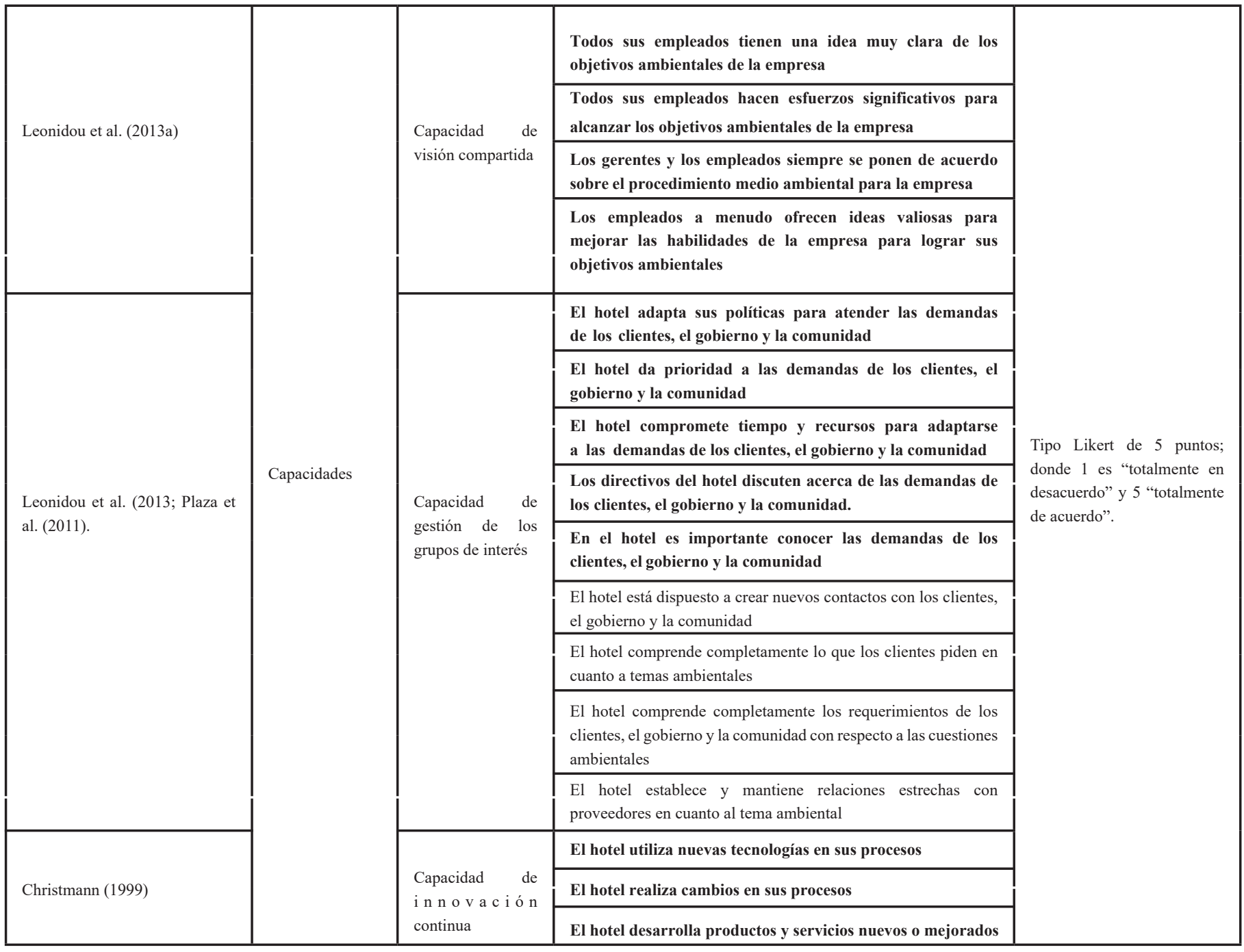




\begin{tabular}{|c|c|c|c|c|}
\hline \multirow{16}{*}{ Leonidou et al. (2013a) } & \multirow{16}{*}{$\begin{array}{l}\text { Estrategia de } \\
\text { mercadotecnia } \\
\text { verde }\end{array}$} & \multirow[t]{4}{*}{ Producto-servicio } & $\begin{array}{l}\text { Utiliza productos ecológicos para la creación del servicio } \\
\text { que ofrece (focos ahorradores de energía, lámparas de } \\
\text { decoración solares, regaderas ahorradoras de agua, } \\
\text { inodoros de doble descarga, sensores de luz en pasillos, } \\
\text { popotes biodegradables, papel higiénico biodegradable, } \\
\text { etc.) } \\
\text { Da preferencia a ofrecer un servicio más respetuoso con el }\end{array}$ & \multirow{16}{*}{$\begin{array}{l}\text { Tipo Likert de } 5 \text { puntos; } \\
\text { donde } 1 \text { es "totalmente en } \\
\text { desacuerdo" y } 5 \text { "totalmente } \\
\text { de acuerdo". }\end{array}$} \\
\hline & & & medio ambiente & \\
\hline & & & $\begin{array}{l}\text { Está orientado a diseñar, desarrollar y ofrecer sus } \\
\text { servicios de una manera respetuosa con el medio ambiente }\end{array}$ & \\
\hline & & & $\begin{array}{l}\text { Ofrece sus servicios de una manera que minimiza su impacto } \\
\text { en el medio ambiente (tarjetas electrónicas de entrada a la } \\
\text { habitación, transporte ecológico, alberca de circuito cerrado que } \\
\text { reutiliza el agua, lámparas solares en jardín, etc.) }\end{array}$ & \\
\hline & & \multirow{4}{*}{ Precio } & $\begin{array}{l}\text { En sus precios incluye los costos de cumplir con el tema } \\
\text { ambiental }\end{array}$ & \\
\hline & & & $\begin{array}{l}\text { Se aprovecha de los ahorros en costos derivados de la utilización } \\
\text { de prácticas respetuosas del medio ambiente, para ofrecer } \\
\text { mejores precios }\end{array}$ & \\
\hline & & & $\begin{array}{l}\text { Aprovecha el éxito financiero de varios productos y/o servicios } \\
\text { respetuosos con el medio ambiente, para reducir sus precios }\end{array}$ & \\
\hline & & & $\begin{array}{l}\text { Ofrece precios competitivos a sus clientes como resultado de las } \\
\text { prácticas ecológicas implementadas }\end{array}$ & \\
\hline & & \multirow{4}{*}{$\begin{array}{l}\text { Plaza } \\
\text { (distribución) }\end{array}$} & $\begin{array}{l}\text { Anima a sus proveedores, vendedores y agentes, y representantes } \\
\text { a adoptar y reflejar la responsabilidad ambiental }\end{array}$ & \\
\hline & & & $\begin{array}{l}\text { Muestra preferencia por proveedores y socios estratégicos que se } \\
\text { encuentran trabajando la responsabilidad ambiental }\end{array}$ & \\
\hline & & & $\begin{array}{l}\text { Su hotel es cuidadoso al elegir suministros y consumibles que } \\
\text { sean respetuosos con el medio ambiente (amenidades, productos } \\
\text { biodegradables para la limpieza, productos biodegradables para } \\
\text { blancos) }\end{array}$ & \\
\hline & & & Compra suministros a granel para reducir el empaque & \\
\hline & & \multirow{4}{*}{ Promoción } & $\begin{array}{l}\text { Destaca su compromiso con la preservación del medio } \\
\text { ambiente en sus anuncios, patrocinios y/o campañas }\end{array}$ & \\
\hline & & & $\begin{array}{l}\text { Realiza esfuerzos promocionales y comunicacionales } \\
\text { donde destacan e informan a sus clientes sobre sus } \\
\text { esfuerzos ambientales }\end{array}$ & \\
\hline & & & Comunica sus iniciativas ambientales a todos los empleados & \\
\hline & & & $\begin{array}{l}\text { Utiliza argumentos ecológicos en sus anuncios, material } \\
\text { promocional y/o campañas de mercadotecnia }\end{array}$ & \\
\hline
\end{tabular}

